\section{Case Reports in Dermatology}

\title{
A Case of Facial Partial Unilateral Lentiginosis Treated with Low-Fluence 1,064 nm Q-Switched Neodymium-Doped Yttrium Aluminum Garnet Laser
}

\author{
En Hyung Kim
}

Department of Dermatology, Cheil General Hospital and Women's Healthcare Center, Dankook University College of Medicine, Cheonan, South Korea

\section{Keywords}

Partial unilateral lentiginosis · 1,064-nm Q-switched neodymium-doped yttrium aluminum garnet laser

\begin{abstract}
Partial unilateral lentiginosis (PUL) is an unusual pigmentary disorder characterized by numerous lentigines grouped within an area of normal skin. Although treatment is not necessary, many patients with facial PUL seek medical help for cosmetic reasons. There is no established standard treatment for PUL. Conventional lasers may cause postinflammatory hyperpigmentation because keratinocytes are injured during the process. Also, scarring, long downtime, and pain are important issues. A 19-year-old patient with facial PUL was successfully treated with low-fluence 1,064-nm Q-switched neodymium-doped yttrium aluminum garnet (QS Nd:YAG) laser. Although the exact mechanism by which low-fluence 1,064-nm QS $\mathrm{Nd}$ :YAG laser improves pigmentary lesions is unclear, the terms "subcellular selective photothermolysis" and "melanocyte apoptosis and replacement" have been proposed. If appropriate measures are taken to monitor patient response during and after the procedure, lowfluence 1,064-nm QS Nd:YAG laser may achieve good cosmetic results in the treatment of PUL with a very safe and effective profile.




\section{Introduction}

Partial unilateral lentiginosis (PUL) is a rare pigmentary disorder characterized by multiple lentigines grouped within an area of normal skin, often in a segmental pattern [1]. In most patients with PUL, the lesions first appear during early childhood (mean age 5 years; range from birth to 15 years) [2]. It is characterized by multiple lentigines that are grouped in a segmental pattern with a sharp demarcation at the midline. PUL can appear anywhere on the body, including the face, neck, trunk, and extremities [2]. Although treatment is not necessary, many patients with facial PUL complain of feeling intimidated during interpersonal relationships because of the cosmetically disfiguring nature of the location. There is no established standard treatment for PUL. Treatments using conventional laser methods are sometimes unsatisfactory due to common side effects, such as postinflammatory hyperpigmentation (PIH), scarring, and recurrences. And as patients with facial PUL who seek treatment are mostly of young age, long downtime and pain are also important issues. Over the last few years, the 1,064-nm Q-switched neodymium-doped yttrium aluminum garnet (QS Nd:YAG) laser has increasingly been used for "laser toning" or "laser facial" as well as for nonablative skin rejuvenation and melasma in Asian countries [3]. In "laser toning," multiple passes of a low-fluence laser (1.6-3.5 J/ $\left.\mathrm{cm}^{2}\right)$ are delivered through a large spot size (6-8 $\mathrm{mm}$ ) to optimize energy delivery. The intention is to have multiple laser treatments at subthreshold fluences to obtain clinical effects with less downtime [4, 5]. Herein, we report a case of facial PUL successfully treated with low-fluence 1,064-nm QS Nd:YAG laser.

\section{Case Report}

A 19-year-old female presented with a history of unilateral hyperpigmented macules on her left cheek and periorbital area since early childhood. Physical examination revealed multiple brown macules measuring 1.5-2.5 mm in diameter with some lesions showing agminated forms. There was no family history of similar pigmented lesions or personal history of neurological problems. Under the clinical impression of facial PUL, the patient was subjected to 10 laser treatment sessions using a 1,064-nm QS Nd:YAG laser (MedLite C6 ${ }^{\mathrm{TM}}$, Hoya ConBio Inc., Fremont, CA, USA) after obtaining written informed consent. The patient was treated at 1-week intervals starting with a spot size of $6 \mathrm{~mm}$, a fluence of $2.5 \mathrm{~J} / \mathrm{cm}^{2}$, and a pulse rate of $10 \mathrm{~Hz}$ with 1-2 passes of slow sliding technique with approximately 5-15\% overlap. The clinical end point was immediate lightening of pigment or mild erythema without petechiae. The fluence was elevated to $3 \mathrm{~J} / \mathrm{cm}^{2}$ after the 4 th session according to treatment response and patient tolerance. Ice packs were immediately applied to the entire face after laser treatment, and the patient was instructed to use broad-spectrum sunscreen daily. Patient photos were taken at baseline (Fig. 1a), after 4 sessions before fluence elevation (Fig. 1b), and 4 weeks after the last treatment session (Fig. 1c). The treatment was well tolerated with no significant side effects, including purpura, PIH, or scarring, except for mild erythema and a transient burning sensation immediately after the laser treatment, which disappeared within $1 \mathrm{~h}$. 


\section{Case Reports in Dermatology}

\section{Discussion}

PUL have been reported under various names, including unilateral lentigines, lentiginous mosaicism, segmental lentiginosis, zosteriform lentiginous naevus, and agminated lentiginosis [3]. There have been reports of PUL in patients having either contralateral or bilateral segmental neurofibromatosis (NF) 1 with both café-au-lait macules and neurofibromas [2]. Also, other cases of PUL, coexisting with ipsilateral Lisch nodules, axillary freckling, or skeletal alterations, seem to suggest that many cases of PUL represent a particular form of segmental NF1 lacking neurofibromas, i.e., a forme fruste of segmental NF1 [6]. So, it would seem prudent to rule out NF1 in patients with PUL. Also, a few cases of PUL were associated with abnormalities of the central nervous system, including mental retardation and ipsilateral cerebrovascular abnormalities with focal epilepsy, leading to the idea that both conditions resulted from a common developmental problem of the neural crest [7]. Consistent with this finding, it has been suggested that chromosomal mosaicism confined to neural crest melanoblasts may have some role in this disorder [7].

Although the exact mechanism by which low-fluence 1,064-nm QS Nd:YAG laser improves pigmentary lesions is unclear, the term "subcellular selective photothermolysis" has been proposed to describe the mechanism of improvement of melasma with low-fluence 1,064-nm QS Nd:YAG laser [8]. It has been proposed that, by delivering repetitive laser energy using a sub-photothermolytic fluence $\left(<5 \mathrm{~J} / \mathrm{cm}^{2}\right)$ over a large spot size, melanin granules are fragmented and dispersed into the cytoplasm without cellular destruction. Because mature melanosomes accumulate in the dendrites of melanocytes, the selective photothermolytic effect of the laser can be focused intensively on dendrites. The volume of the melanocytes, the number of melanosomes, and the connecting melanocytic dendrites in individuals with melasma are reduced significantly in the epidermis with laser treatment. Therefore, this result can also be interpreted as a selective photothermolytic effect of the laser on mature melanosomes. These results revealed that low-fluence QS Nd:YAG laser treatment had a subcellular selective photothermolytic effect on a subcellular-specific organelle: melanin [3, 8]. Another explanation is melanocyte apoptosis and replacement [9]. The weekly laser treatment destroys melanocytes completely and accelerates apoptotic melanocyte cell death, thus removing abnormal epidermal melanocytes [9-12]. Abnormal melanocytes are replaced by normal melanocytes which migrate from outer root sheath of hair [9-12].

The mechanism of low-fluence 1,064-nm QS Nd:YAG laser treatment is to minimize epidermal damage and destroy melanosomes in the epidermal melanocytes. Conventional highfluence laser techniques may cause PIH because keratinocytes are injured during the process. $532 \mathrm{~nm}$ of QS Nd:YAG laser, $694 \mathrm{~nm}$ of ruby laser, $755 \mathrm{~nm}$ of alexandrite, and 515-755 $\mathrm{nm}$ of intense pulsed light (IPL) absorb much more melanin than 1,064 nm of QS Nd:YAG laser. Thus, fluence that destroys epidermal melanocytes injures the surrounding keratinocytes, and the damaged keratinocytes secrete endothelin-1, $\alpha-\mathrm{MSH}, \mathrm{ACTH}$, prostaglandin (PGE2, PGF2 $\alpha$ ), and nitric oxide. These cytokines activate melanocytes and increase melanin synthesis in the melanosomes, therefore causing PIH [10-12]. Free radical oxygen and peroxide from the keratinocytes also activate melanocytes and increase melanin synthesis in the melanosomes, eventually causing PIH [10-12]. Conventional high-fluence laser treatments cause petechiae and crusts, which may result in damage to fibroblasts, mast cells, lymphocytes, macrophages, and vascular endothelium. Human fibroblasts secrete several melanogenic cytokines, such as bFGF, HGF, and SCF, when they are rapidly growing or during inflammation, which suggests the possibility that overexpression of these cytokines by dermal fibroblasts may activate melanocytes in the overlying epidermis [13]. It is also possi- 
ble that the resulting metabolites from the arachidonic acid pathway and histamine from mast cells may affect melanogenesis [14], thus resulting in PIH.

During the laser procedure, attention should be paid not to perform multiple passes of laser by a sliding-stacking technique as epidermal damages may occur and the damaged keratinocytes might secrete cytokines, such as endothelin-1, $\alpha$-MSH, ACTH, bFGF, prostaglandin (PGE2, PGF2 $\alpha$ ), and nitric oxide, which could cause PIH. Although our patient's treatment outcome was satisfactory, recurrence may also be an issue as low-fluence treatment for melasma has shown recurrence. But as treatment was well tolerated without PIH or aggravation of lesions, recurrence may be treated with the same method. Although not noted in our patient, mottled hypopigmentation has been reported after multiple sessions of low-fluence 1,064-nm QS Nd:YAG laser treatment for melasma [15]. Even though these lesions are reversible after termination of treatment, they could be disfiguring and stressful to the patient. So, precaution should be taken when elevating laser fluence, and for some patients, even when low sub-photothermolytic fluences are used for treatment, the total cumulative dose after multiple treatment sessions may exceed the total toxic cumulative energy that would destroy the melanocytes [3]. Careful monitoring of patient response may prevent such side effects.

Low-fluence 1,064-nm QS Nd:YAG laser treatment does not provoke side effects, such as petechiae, crusts, pain, and PIH, during the laser treatment. It may achieve good cosmetic results in the treatment of PUL with a very safe and effective profile.

\section{Statement of Ethics}

Informed consent was obtained from the patient for being included in this case report.

\section{Disclosure Statement}

The authors confirm that there are no conflicts of interest.

\section{References}

1 Thompson GW, Diehl AK: Partial unilateral lentiginosis. Arch Dermatol 1980;116:356.

Schaffer JV, Lazova R, Bolognia JL: Partial unilateral lentiginosis with ocular involvement. J Am Acad Dermatol 2001;44;387-390.

-3 Lee YH, Choi EH, Lee SW: Low-fluence Q-switched 1,064-nm neodymium-doped yttrium aluminum garnet laser for the treatment of facial partial unilateral lentiginosis in Koreans. Dermatol Surg 2012;38:31-37.

4 Jeong SY, Shin JB, Yeo UC, Kim WS, et al: Low-fluence Q-switched neodymium-doped yttrium aluminum garnet laser for melasma with pre- or post-treatment triple combination cream. Dermatol Surg 2010;36:909-918.

-5 Polnikorn N: Treatment of refractory dermal melasma with the MedLite C6 Q-switched Nd:YAG laser: two case reports. J Cosmet Laser Ther 2008;10:167-173.

6 Chen WC, Fan PC, Happle R: Partial unilateral lentiginosis with ipsilateral Lisch nodules and axillary freckling. Dermatology 2004;209:321-324.

7 Pickering JG: Partial unilateral lentiginosis with associated developmental abnormalities. Guys Hosp Rep 1973;122:361-370.

8 Mun JY, Jeong SY, Kim JH, Han SS, et al: A low fluence Q-switched Nd:YAG laser modifies the 3D structure of melanocyte and ultrastructure of melanosome by subcellular-selective photothermolysis. J Electron Microsc (Tokyo) 2011;60:11-18. 


\section{Case Reports in Dermatology}

\begin{tabular}{l|l}
\hline Case Rep Dermatol 2017;9:30-34 \\
\hline DOI: 10.1159/000477376 & $\begin{array}{l}\text { C 2017 The Author(s). Published by S. Karger AG, Basel } \\
\text { www.karger.com/cde }\end{array}$ \\
\hline
\end{tabular}

Kim: A Case of Facial Partial Unilateral Lentiginosis Treated with Low-Fluence 1,064 nm Q-Switched Neodymium-Doped Yttrium Aluminum Garnet Laser

Hur H, Kim YR, Shim DT: The treatment of café au lait spot, partial unilateral lentiginosis and Becker's nevus using a high fluence 1,064-nm Q-switched Nd:Yag laser. J Clin Cosmet Dermatol 2017, DOI: http://dx.doi.org/10.16966/jccd.111.

10 Hattori H, Kawashima M, Ichikawa Y, Imokawa G: The epidermal stem cell factor is over-expressed in lentigo senilis: implication for the mechanism of hyperpigmentation. J Invest Dermatol 2004;122:1256-1265.

11 Okazaki M, Yoshimura K, Suzuki Y, Uchida G, Kitano Y, et al: The mechanism of epidermal hyperpigmentation in café-au-lait macules of neurofibromatosis type 1 (von Recklinghausen's disease) may be associated with dermal fibroblast-derived stem cell factor and hepatocyte growth factor. Br J Dermatol 2003;148:689-697.

12 Okazaki M, Youshimura K, Uchida G, Suzuki Y, Kitano Y, et al: Epidermal hyperpigmentation in nonsyndromic solitary café-au-lait macules may be associated with increased secretion of endothelin-1 by lesional keratinocytes. Scand J Plast Reconstr Surg Hand Surg 2005;39:213-217.

13 Imokawa G: Autocrine and paracrine regulation of melanocytes in human skin and in pigmentary disorders. Pigment Cell Res 2004;17:96-110.

14 Abdel-Malek Z, Kadekaro AL: Human pigmentation: its regulation by ultraviolet light and by endocrine, paracrine, and autocrine factors; in Nordlund JJ, Boissy RE, Hearing VJ, King RA, Oetting WS, Ortonne JP (eds): The Pigmentary System. Oxford, Blackwell Publishing Ltd, 2006, pp 410-420.

15 Kim MJ, Kim JS, Cho SB: Punctate leucoderma after melasma treatment using 1,064-nm Q-switched Nd:YAG laser with low pulse energy. J Eur Acad Dermatol Venereol 2009;23:960-962.
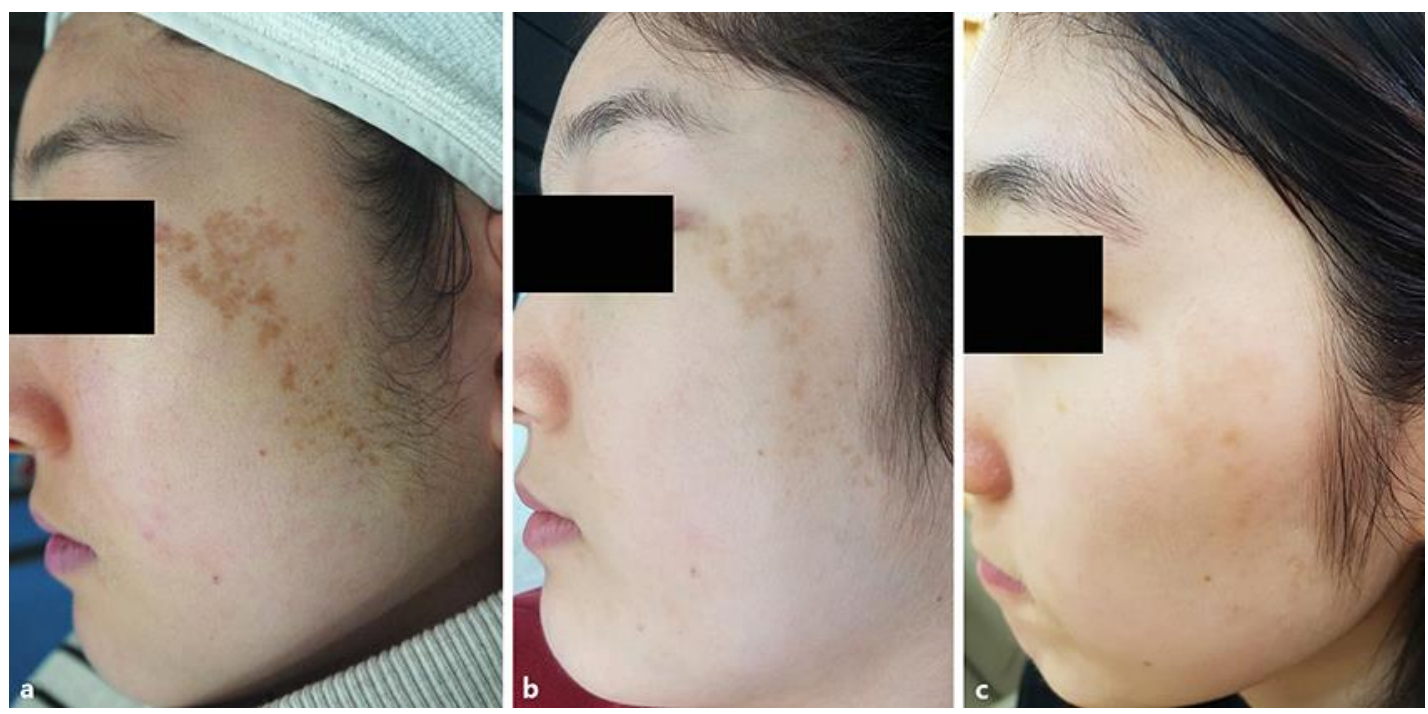

Fig. 1. Clinical manifestations and treatment response of the patient. Before treatment (a), after 4 treatment sessions before fluence elevation (b), and 4 weeks after completion of laser treatment (c) 